Methods Using comparable datasets from a local community in South East London during 2008-2010 ( $\mathrm{n}=1,698)$ and from England in 2007 ( $n=7,403)$, latent class analysis was used to construct profiles of polydrug users. Eight binary indices reporting drug use in the last year for cannabis, amphetamine, cocaine, ecstasy, LSD, tranquilisers, crack, heroin, and two items for hazardous alcohol drinking and current cigarette smoking, were regressed on a latent variable of polydrug use with age and sex covariates. The latent class solution from each dataset was then used in a multinomial logistic regression comparing risk factors from socio-demographic, neighbourhood, social support, and mental health domains.

Results Both datasets produced three class solutions with comparable proportions: in London, 61.6\% were in the "Nondrug user" group, $30.5 \%$ in the "Moderate user group" (characterised by hazardous drinking, cigarettes and some cannabis use) and 7.9\% in the "High Drug User" group (characterised by drug use across all substances); in the national dataset the proportions were $57.4 \%, 37.9$ $\%$, and $4.6 \%$ respectively. In a logistic regression comparing both polydrug user groups to the nonuser group, both samples reported higher odds for polydrug use and common mental disorder, suicidal ideation, and functional limitation; higher levels of education, stressful life events and a never-married status were also associated with polydrug use. Differences between the local and national samples for polydrug use were found on factors such as ethnicity, social support, and employment status. Further analysis revealed no difference between the "Moderate" and "High Drug" groups in the local sample for these risk factors and mental health indicators, whereas several dose-response relationships between these groups were found nationally.

Conclusion Approximately a third of the general public both nationally and locally exhibit a pattern of moderate polydrug use associated with mental health and daily functioning impairments. Furthermore, local services and policy makers should note that higher education is a risk factor for polydrug use, and that factors related to employment and social support may be differentially linked to substance use depending on geographical residence.

\section{PS41 CHARACTERISTICS ASSOCIATED WITH STATIN PRESCRIBING FOR PRIMARY PREVENTION OF CARDIOVASCULAR DISEASE AMONG PEOPLE WITH DIABETES}

doi:10.1136/jech-2012-201753.140

NRV Jones, SH Wild. Centre for Population Health Sciences, University of Edinburgh, Edinburgh, UK

Background Diabetes is a risk factor for cardiovascular disease (CVD) and guidelines recommend that people with diabetes ought to have their blood lipid levels monitored and, if necessary, controlled to ensure that they are at a safe level to prevent avoidable morbidity and mortality resulting from CVD. Guidelines are not necessarily followed and the differences in treatment which this leads to could give rise to health inequalities according to socioeconomic status, sex, or other patient characteristics. This study investigated the association between patient characteristics and statin prescribing in people who were eligible to receive a statin for primary prevention of CVD according to the contemporary Scottish Intercollegiate Guideline Network guidelines (SIGN 55).

Methods Data from the Scottish Care Information - Diabetes Collaboration dataset for the period 2000-2007 were used. This dataset contains socio-demographic and prescribing data for 203,528 people, which is almost every person with diagnosed diabetes in Scotland. The analyses were based on people over 40 years of age, with complete data, with no history of CVD, and with total serum cholesterol exceeding $5 \mathrm{mmol} / 1$.

Logistic regression was used to calculate odds ratios (OR) for ever having a record of a statin prescription by age, sex, socioeconomic status (defined using quintiles of the Scottish Index of
Multiple Deprivation, SIMD, where Q1 reflects the most deprived and $\mathrm{O} 5$ the most affluent), smoking habits, body mass index, diastolic blood pressure, and type of diabetes.

Results Of 83,666 people identified as eligible for statin treatment as defined by SIGN 55 guidelines, $29 \%$ had no record of a statin prescription. In both men and women, the OR for having a statin prescribed when compared to Q1 from multi-variate models were $\mathrm{OR}_{\text {men }} 0.93 \mathrm{OR}_{\text {women }} 0.91$ for $\mathrm{Q} 2, \mathrm{OR}_{\text {men }} 0.77 \mathrm{OR}_{\text {women }} 0.77$ for $\mathrm{O} 3$, and $\mathrm{OR}_{\text {men }}^{\text {men }} 0.71 \mathrm{OR}_{\text {women }} 0.71$ for $\mathrm{Q} 4$. Current and former smokers, overweight and obese people, and people with high blood pressure or treated hypertension had greater odds of statin prescription, whereas underweight people and women with type one diabetes had reduced odds of being prescribed a statin than each comparison group.

Conclusion Almost one third of people with diagnosed diabetes have no record of having ever received a statin prescription as recommended by SIGN 55. The odds of having a record of treatment were higher among more deprived people even after adjusting for potential confounding factors.

\section{PS42 INEQUALITIES IN CHILD OBESITY: WHERE DO THESE OCCUR AND IS THE GAP WIDENING?}

doi:10.1136/jech-2012-201753.141

H Dinsdale, C Ridler. Health Intelligence, National Obesity Observatory, Oxford, UK

Background Child obesity is an important public health problem. Of particular concern are the considerable inequalities in obesity prevalence between socioeconomic and ethnic groups. The Department of Health's recently published Call to Action on Obesity makes a commitment to achieving a sustained downward trend in the level of excess weight in children and states that it is vital that action on obesity reduces health inequalities.

Methods This analysis uses data collected by the National Child Measurement Programme (NCMP). The NCMP is an annual programme that measures the height and weight of children aged 4-5 years (Reception) and 10-11 years (Year 6) in schools in England. Approximately one million children are measured every year.

The NCMP collects information on ethnicity and place of residence for each child. Five years of good quality data are now available (2006/07, 2007/08, 2008/09, 2009/10, 2010/11) and these have been analysed in detail by the National Obesity Observatory to examine how patterns of child obesity prevalence vary by demographic and socioeconomic group.

Results Obesity prevalence among children who live in the most deprived areas of England is approximately twice that of children living in the least deprived areas. NCMP data suggest that health inequalities among boys in Reception and girls in Year 6 are widening. Health inequalities do not seem to be widening or narrowing for girls in Reception or boys in Year 6 but substantial health inequalities do persist among these groups.

When all years of NCMP measurements are considered the Bangladeshi ethnic group seems to have shown the greatest increases over time. Children in the 'White Other' ethnic group appear to be experiencing a decrease in obesity prevalence.

Conclusion In order to achieve a reduction in obesity prevalence among children a particular focus on deprived groups may be required. This would help tackle persistent health inequalities. Children from the Bangladeshi ethnic group may benefit from particular attention, given the evidence that obesity prevalence is increasing for these children at a greater rate than for other ethnic groups.

PS43 WHAT IS IMPORTANT TO THE QUALITY OF LIFE OF PEOPLE WITH MULTIPLE MYELOMA? IMPLICATIONS FOR THE DESIGN OF OUALITY OF LIFE QUESTIONNAIRES

doi:10.1136/jech-2012-201753.142 